\title{
Article \\ Detecting Criticality by Exploring the Acoustic Activity in Terms of the "Natural-Time" Concept
}

\author{
Andronikos Loukidis ${ }^{1}$ (D), Dimos Triantis ${ }^{1, *(\mathbb{D})}$, Ilias Stavrakas ${ }^{1}$ (D), Ermioni D. Pasiou ${ }^{2}$ (D) \\ and Stavros K. Kourkoulis 2 (D)
}

1 Electronic Devices and Materials Laboratory, Department of Electrical \& Electronics Engineering, University of West Attica, 250 Thivon Avenue, 12244 Athens, Greece; a.loukidis@uniwa.gr (A.L.); ilias@uniwa.gr (I.S.)

2 Laboratory for Testing and Materials, Department of Mechanics, School of Applied Mathematical and Physical Sciences, Zografou Campus, National Technical University of Athens, 15773 Athens, Greece; epasiou@teemail.gr (E.D.P.); stakkour@central.ntua.gr (S.K.K.)

* Correspondence: triantis@uniwa.gr; Tel.: +30-2105381316

Citation: Loukidis, A.; Triantis, D.; Stavrakas, I.; Pasiou, E.D.;

Kourkoulis, S.K. Detecting Criticality by Exploring the Acoustic Activity in Terms of the "Natural-Time" Concept. Appl. Sci. 2022, 12, 231. https:// doi.org/10.3390/app12010231

Academic Editor: Dario De Domenico

Received: 6 December 2021

Accepted: 23 December 2021

Published: 27 December 2021

Publisher's Note: MDPI stays neutral with regard to jurisdictional claims in published maps and institutional affiliations.

Copyright: (C) 2021 by the authors. Licensee MDPI, Basel, Switzerland. This article is an open access article distributed under the terms and conditions of the Creative Commons Attribution (CC BY) license (https:// creativecommons.org/licenses/by/ $4.0 /)$.

\begin{abstract}
The acoustic activity developed in marble specimens under various loading schemes is explored in terms of the recently introduced F-function. The novelty of the study is that instead of describing the temporal evolution of the F-function in terms of conventional time, the Natural Time concept is employed. Although completely different geometries and loading schemes were considered, the evolution of the F-function in the Natural Time domain exhibits a self-consistent motive: its values increase progressively with fluctuations of varying intensity, however, while the fracture is approaching, a power law appears to systematically govern the response of the specimen/structure loaded. The exponent of this law, somehow corresponding to the intensity of the acoustic activity within the loaded complex, varies within broad limits. The onset of validity of the power law designates that the system has entered into its critical stage, namely that of impending fracture, providing a useful pre-failure signal.
\end{abstract}

Keywords: acoustic emissions; natural time; pre-failure indicators; marble; F-function

\section{Introduction}

The irreversible processes (for example, microcracking, propagation and coalescence of microcracks, various changes of the microstructure) taking place due to the activation of damage mechanisms at the interior of loaded structural elements are responsible for the generation of acoustic emissions (AEs). The respective sensing technique that detects and records these emissions, namely the AE technique, was introduced long ago [1-3] and is still under further development. Gradually it became an extremely useful tool in the hands of engineers from various disciplines since it allows the real-time monitoring of the damage evolution under either quasi-static or dynamic loads, as well as the characterization/classification of the failure modes taking place [4]. Nowadays, the AE technique is considered the most mature and reliable tool in the attempt to understand the mechanisms activated before the loaded structure enters into its critical stage (i.e., that of impending fracture) and during this critical stage. Moreover, it is widely used for structural health monitoring purposes.

Quite a few characteristics of the AEs (such as, for example, their rate of occurrence, their cumulative energy, the temporal evolution of b-values, the cumulative number of counts, etc.) are properly elaborated statistically enlightening interesting aspects of the overall response of the loaded structures [4-9]. The number of hits recorded per second is an often-used index for monitoring the acoustic activity during a loading/fracture process. Recently an alternative procedure was introduced, which offers monitoring the acoustic activity with increased resolution by taking advantage of the inter-event time 
intervals between two successive hits. The procedure is based on the F-function [10], which is determined by utilizing the interevent time intervals of a sufficient number of $n$ ( $n>$ 10) consecutive AE hits/events. In fact, the $F$-function represents the average frequency of appearance of AE hits/events in a sliding time window including $n$ consecutive AE hits/events. Each value of the $F$-function is paired to an average time instant, $\tau$, determined as the average of the time instants at which each one of the $n$ successive hits of the group contained in the specific time window appeared.

Contrary to the traditional way of representing the AE activity through the "numberof-hits-per-second", the temporal evolution of the F-function is advantageous since it highlights according to a clearer manner the variability of the acoustic activity, especially during the last phase of the loading process just before the catastrophic fracture. Taking into account that the vast majority of acoustic events are recorded during the very last time instants prior to the macroscopically observed catastrophic fracture, the time evolution of the F-function is usually described in terms of the "time-to-failure" parameter, $\left(t_{f}-\right.$ $\tau$ ), where $t_{f}$ is the time instant when the last AE hit/event was recorded (i.e., the time instant of the catastrophic fracture, when usually an AE hit/event with an amplitude of $99 \mathrm{~dB}$ is detected). Adopting the specific description offers an enlightening view of what happens during the very last loading instants. The use of the $F$-function for the exploration of acoustic activity is nowadays adopted by many researchers worldwide [11-17].

The temporal evolution of the F-function in terms of the "time-to-failure" parameter, during the last loading stages before the catastrophic fracture, was found to obey a power law $[10,18]$ of the form:

$$
F=A \cdot\left(t_{f}-\tau\right)^{-m}
$$

where $A$ is a constant and $m$ is an exponent, the numerical value of which ranges around the unit. In general, the exponent $m$ characterizes the intensity of the rate of production of acoustic hits/events just before fracture. Moreover, it has been experimentally verified $[19,20]$ that the temporal evolution of the $F$-function provides indices that could be safely considered as pre-failure signals.

In this direction, and in an attempt to detect complementary pre-failure indicators, the evolution of the F-function is considered here in terms of the Natural Time, $\chi$, rather than in terms of conventional time (i.e., of the "time-to-failure" parameter). The onset of validity of the power-law of Equation (1) is indicative of the (self-organized) criticality, which governs the response of the specimens, just before catastrophic fracture. According to the influential work of Bak et al. (1987) [21], various complex systems, operating far from their thermodynamic equilibrium, will be spontaneously organized under their natural evolution (and without the need of an external intervention), into a critical boundary state which resides at the transition point between the equilibrium and non-equilibrium states. During this phase transition, the appearance of power (or approximately power) laws has been extensively reported in the literature $[9,19]$.

Within these lines and considering that the generation of AEs while fracture approaches is a non-equilibrium phenomenon of complex dynamic systems (namely the brittle specimens under severe mechanical load), the Natural Time concept has been proven as a quite flexible alternative theoretical framework for describing the proximity of the system to criticality (i.e., to catastrophic fracture) [22,23], which in the case of the F-function is manifested through the power-law of Equation (1).

The analysis of the acoustic emission data, recorded during a series of experimental protocols with marble specimens, revealed definitely that a power law governs, again, the evolution of the F-function in terms of Natural Time during the intervals that the load-carrying capacity of the loaded element tends to be exhausted. The instant at which this power law starts governing the mechanical response of the system reflects somehow a change of the damage mechanisms which are activated within the specimen and these mechanisms that will lead to the macroscopic destruction of the specimen start prevailing. Thus, the onset of validity of this power law may be considered as the threshold beyond 
which the system enters into its critical stage (i.e., the stage of impending fracture) providing a pre-failure signal, which deserves to be further investigated.

\section{Theoretical Preliminaries}

The concept of Natural Time, $\chi$, was introduced in 2001 by P. Varotsos et al. [24,25] and it was initially used in the field of Seismology. Since then the analysis in the Natural Time domain has been proven quite advantageous when describing the dynamic evolution of complex systems: It reduces uncertainty, allowing optimal extraction of signal information, especially for signals originating from dynamic systems approaching the criticality [26]. In this context, the Natural Time concept became gradually a valuable alternative tool in the tedious effort of the scientific community to develop theories for the prediction of upcoming catastrophic events. Soon after the concept was introduced it was adopted for data analysis in an amazingly wide range of scientific disciplines, from seismology [26-30], to medicine [31,32], condensed matter physics [33] and mechanics of materials [34-40].

As mentioned above in a fracture process, the function $F(\tau)$ is created by assuming that is a time series consisting of $N$ values, namely: $F_{1}=F\left(\tau_{1}\right), F_{2}=F\left(\tau_{2}\right), \ldots, F_{k}=F\left(\tau_{k}\right)$, $\ldots, F_{N}=F\left(\tau_{N}\right)$. Instead of the conventional time $\tau$, a value $k$ of order $(1,2,3 \ldots N)$ will be characterized by the evolution of the pair $\left(\chi_{k}, F_{k}\right)$ where $\chi_{k}=k / N$ and $F_{k}$ is the value of $F\left(\tau_{k}\right)$. This creates the time series $F(\chi)$ where $\chi$ is the Natural Time (see Figure 1 ).
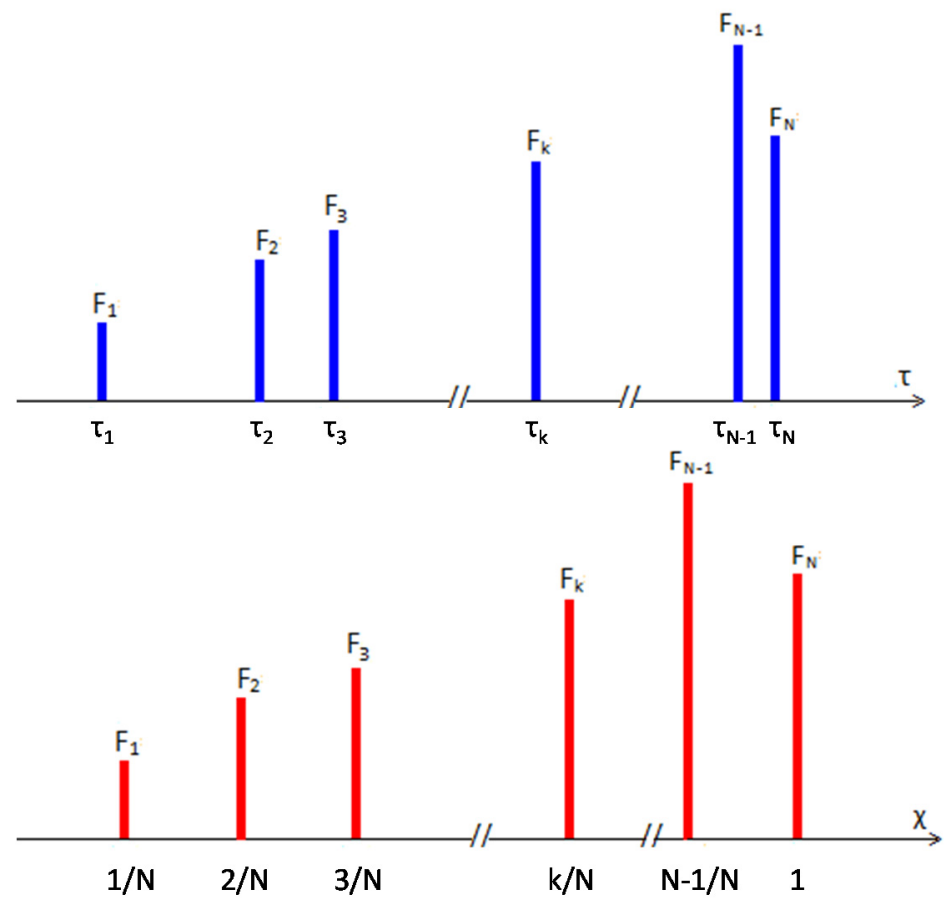

Figure 1. Schematic interpretation of the F-function in terms of conventional time (up) and in the Natural Time domain (down).

In order to further clarify the above-described procedure, a detailed example will be discussed in this section, indicating the way of representing the acoustic activity (expressed here as the rate of occurrence of the acoustic hits) in terms of the F-function, both in the conventional $(\tau)$ and the Natural Time $(\chi)$ domains: a uniaxial tensile test with a Double Edge Notched (DENT) marble specimen (Figure 2a) with notch length equal to $\alpha=8$ $\mathrm{cm}$ is considered. Details of the experimental protocol concerning the specimen and the experimental set-up have been reported in previous works [41,42]. During the specific test, a total of 137 acoustic hits of amplitude $A \geq 40 \mathrm{~dB}$ were recorded, the vast majority of which were detected at the very last loading stages, i.e., when the externally applied load was approaching the tensile strength of the specimen. 

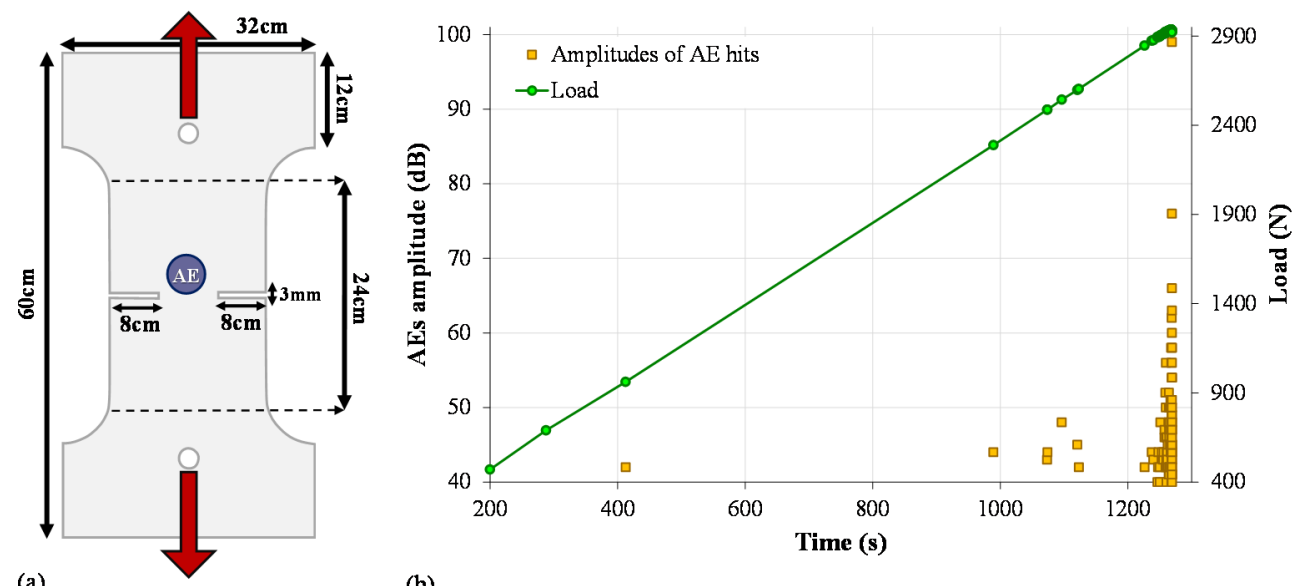

(b)

Figure 2. (a) Geometrical characteristics of the DENT specimen. The red arrows indicate the direction of the imposed tensile force. (b) The temporal evolution of the amplitudes of the acoustic hits in juxtaposition to that of the tensile force.

For the calculation of the $F$-function $n=10$ consecutive inter-event time intervals were used and its evolution in conventional time $\tau$ is presented in Figure 3a. The sharp increase of the $F$-function starts at the instant $\tau \approx 1245 \mathrm{~s}$ when the externally applied tensile force attained a value equal to about $2889 \mathrm{~N}$, corresponding to $98.4 \%$ of the maximum force attained during the specific test. It is clearly seen that densely packed information is hidden in a very narrow time interval (just before fracture) and thus it is quite possible that critical details are lost. Therefore, the need for an alternative representation of the acoustic activity becomes a pressing demand. In this direction, the evolution of the $F$-function is considered in the Natural Time domain $(\chi)$ as it is shown in Figure 3b. It is highlighted that, for the whole time interval for which the applied force varies around its maximum value (i.e., the catastrophic fracture is impending), the function $F(\chi)$ is governed by a power law of the form:

$$
F(\chi)=A \cdot \chi^{m}
$$

where $A$ is a constant $[A=F(\chi=1)]$ and $m$ is an exponent related to how strongly the acoustic activity is intensified at the time instants just before macroscopic fracture.

For the specific experiment analyzed in this section and for the time interval corresponding to the shaded rectangular in Figure $3 \mathrm{~b}$, proper curve fitting yields for $m$ a value equal to 4.65. The interval of $\chi$-values, for which the power law of Equation (2) governs the response of the material starts at $\chi=0.36$ and ends at $\chi=0.85$. The respective values for the applied load are equal to $2931 \mathrm{~N}$ and $2928 \mathrm{~N}$. The maximum force attained was equal to $2933 \mathrm{~N}$, indicating that the power law of Equation (2) governs the response of the material a little before and a little after the maximum force is attained. Translating the above numerical values to the conventional time domain, it can be seen that the power law is valid in the time interval between $2.4 \mathrm{~s}$ and $0.08 \mathrm{~s}$ before fracture (in practical terms until the final fracture of the specimen).

In the sections following, the above-described approach for representing the $F$-function in the Natural Time domain $(\chi)$ will be applied for the exploration of the acoustic activity in a series of characteristic experimental protocols, with Dionysos marble specimens of various geometries subjected to various loading schemes. The composition and physicochemical properties of Dionysos marble are well documented in the international literature. A comprehensive review is provided by Perdikatsis et al. [43] in terms of trace-, isotope- and mineralogical analysis. 

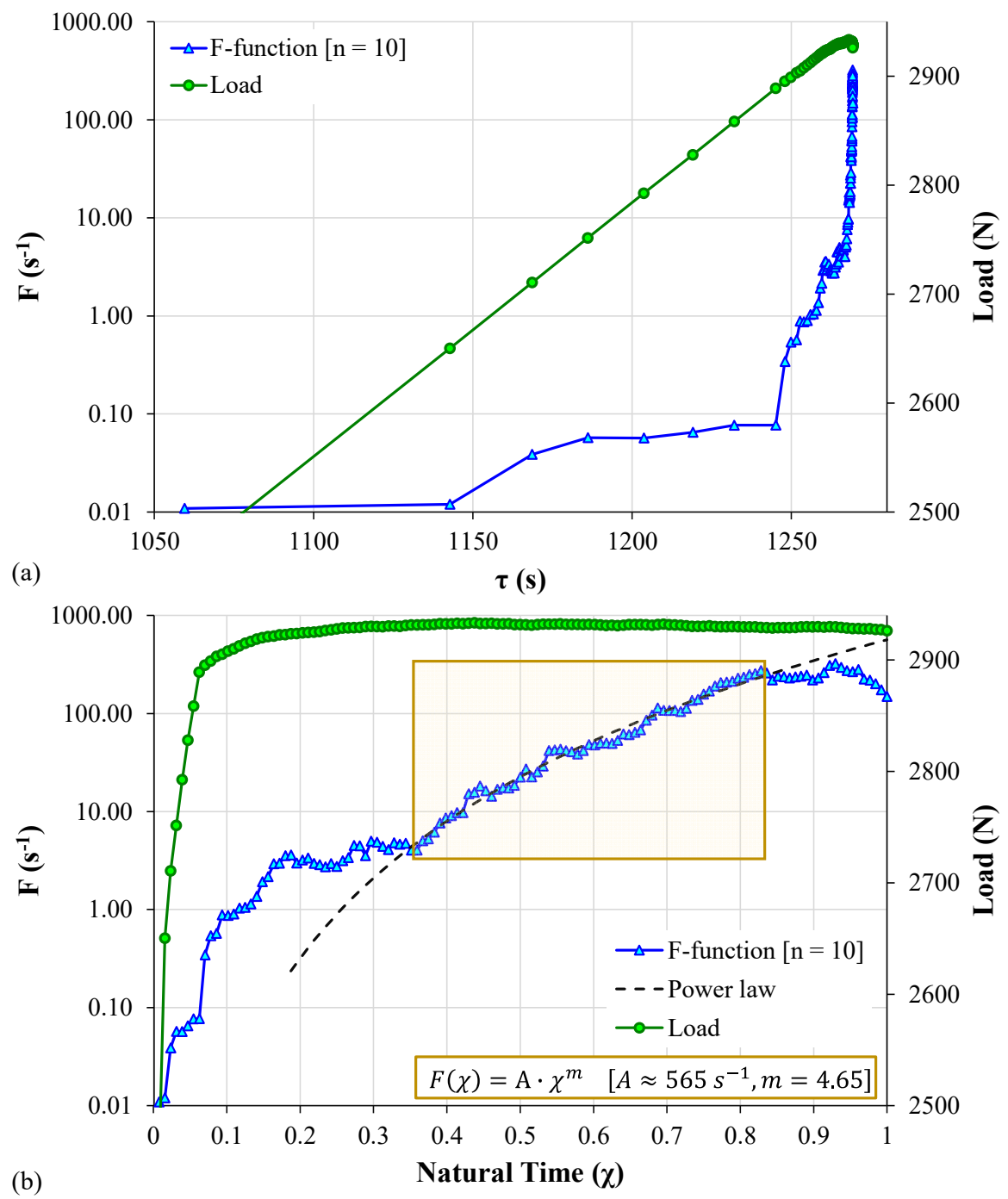

Figure 3. (a) The temporal evolution of the F-function and the load imposed. (b) The respective evolution in the Natural Time domain for the DENT marble specimen.

\section{Analysis of the Acoustic Activity by Means of the F-Function in the Natural Time Domain}

3.1. Three-Point Bending of Notched Marble Prismatic Specimen

Prismatic Dionysos marble specimens (dimensions $20 \mathrm{~mm} \times 20 \mathrm{~mm} \times 100 \mathrm{~mm}$ ) with a central notch (machined mechanically normal to the longitudinal axis of the specimens) were subjected to three-point bending (Figure 4a). The length (depth) of the notch was equal to $\alpha=4 \mathrm{~mm}$ while its width (breadth) was equal to $b=2.5 \mathrm{~mm}$. An AE sensor was attached to the specimen in the immediate vicinity of the crown of the notch (blue circle in Figure 4a) for the detection of acoustic activity. The load was imposed with the aid of a metallic cylinder of radius equal to $10 \mathrm{~mm}$ uniformly all along the width of the specimens and normally to their horizontal axis. Displacement control loading mode was adopted at a constant rate of $0.01 \mathrm{~mm} / \mathrm{min}$, so that quasi-static conditions were simulated. During the experiment, the force applied and the deflection of the central section was recorded together with the characteristics of the AEs. 


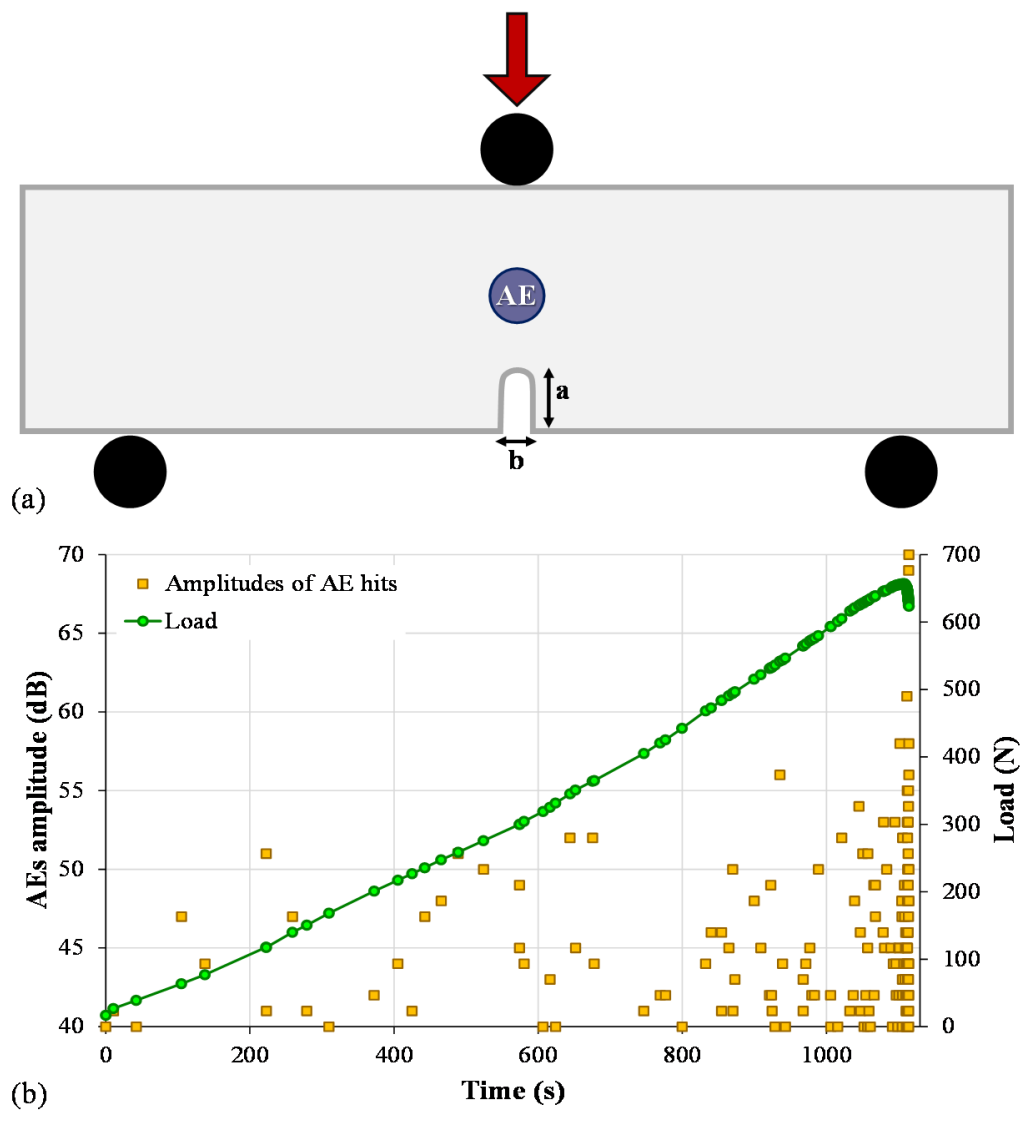

Figure 4. (a) Sketch of the notched prismatic specimen. The red arrow indicates the direction of the imposed load, and the black circles represent the cylinders used to apply the load. (b) The temporal evolution of the amplitudes of the acoustic hits in juxtaposition to that of the force imposed.

Focusing now on a typical experiment of this protocol, $N=176$ acoustic hits of amplitude exceeding $40 \mathrm{~dB}$ were recorded up to the fracture of the specimen. Figure $4 \mathrm{~b}$ shows the time evolution of the load applied and that of the amplitudes of the AEs recorded. The maximum value attained by the force applied was equal to $656.9 \mathrm{~N}$. For the calculation of the $F$-function $n=10$ consecutive interevent time intervals were used.

Its evolution in Natural Time $\chi$ is shown in Figure 5 in juxtaposition to the respective evolution of the load applied. The $F$-function attains a maximum value equal to $38.4 \mathrm{~s}^{-1}$ shortly before the fracture of the specimen. The specific time instant corresponds to about $1.5 \mathrm{~s}$ in conventional time units and about $\chi=0.79$ in the Natural Time domain. The interval within which $F(\chi)$ is governed by the power law of Equation (2), lies in the range from $\chi=0.46$ to $\chi=0.79$ in Natural Time terms. Proper curve-fitting gives for the exponent $m$ (of Equation (2)) a value equal to 8.80. In the interval of validity of Equation (2) the load attained values ranging from $647 \mathrm{~N}$ (before its maximization at $656.9 \mathrm{~N}$ ) to $643 \mathrm{~N}$ after its maximization. 


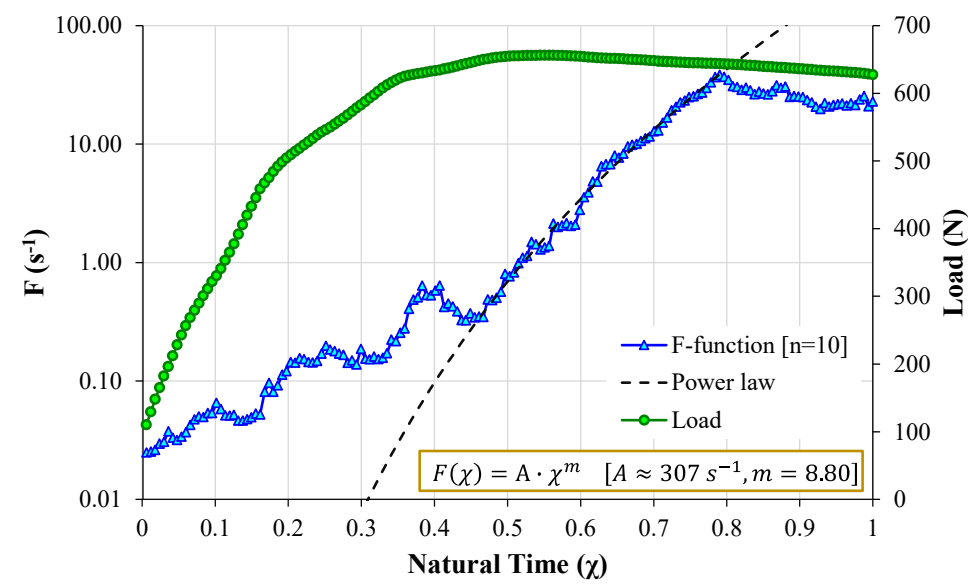

Figure 5. The evolution in the Natural Time domain of the F-function and the load imposed for the notched specimens under three-point bending.

\subsection{Uniaxial Compression of Intact Prismatic Marble Specimen}

Intact prismatic Dionysos marble specimens (dimensions $45 \mathrm{~mm} \times 45 \mathrm{~mm} \times 100 \mathrm{~mm}$ ) were subjected to uniaxial compression. A strain gauge was attached at the center of one of the specimens' lateral surfaces in order to measure the axial strain developed, while an acoustic sensor was attached at the center of the adjacent lateral surface (Figure 6a). The compressive force was applied at a constant rate equal to $475 \mathrm{~N} / \mathrm{s}$. During the experiments, the force and the strain developed were recorded at a sampling rate of 75 values per minute. In addition, the acoustic signals of amplitude $A \geq 45 \mathrm{~dB}$ were recorded. The time evolution of their amplitudes is shown in Figure $6 b$ together with the respective evolution of the force applied. The axial stress-axial strain $(\sigma-\varepsilon)$ curve for a typical experiment of the protocol is shown in Figure 7a. The linear relationship, expected for brittle rocks, characterizes the major portion of the $\sigma-\varepsilon$ curve, corresponding to Young's modulus equal to about $70 \mathrm{GPa}$ (the initial non-linearity for $t<7 \mathrm{~s}$ is attributed to unavoidable bedding errors). The time evolution of the axial strain is shown in Figure $7 \mathrm{~b}$ together with the respective linear fitting, which governs the response of the material up to the $t=181 \mathrm{~s}$ time instant. At this time instant, the stress level attained is equal to about $84 \mathrm{MPa}$, which corresponds to about $88 \%$ of the specimen's compressive strength. For the specific experiment more than 1000 acoustic hits were recorded until the fracture of the specimen, most of which (more than $50 \%$ ) were recorded after the specimen abandoned its linear response. Taking into account the overall number of hits recorded the sliding window used for the calculation of the $F$-function included $n=50$ consecutive hits.

The evolution of the $F$-function in Natural Time $\chi$ is shown in Figure 8 in juxtaposition to the respective evolution of the axial strain recorded. In the same figure, the linear fit of the axial strain in Natural Time terms is plotted. It is clearly seen from this figure that, as soon as the material ceases responding linearly, the evolution of the $F$-function in the Natural Time domain is, again, governed by the power law of Equation (2).

Proper fitting of the data gives the exponent $m$ a value equal to $m=5.26$. The range of applicability of the power law covers the interval from $\chi=0.58$ to $\chi=0.93$. The corresponding conventional-time interval extends from $t=189 \mathrm{~s}$ to $t=198 \mathrm{~s}$, while the corresponding values of the axial stress are $89.9 \mathrm{MPa}$ and $94.2 \mathrm{MPa}$, respectively, or, equivalently, $94 \%$ and $99 \%$ of the material's compressive strength. 


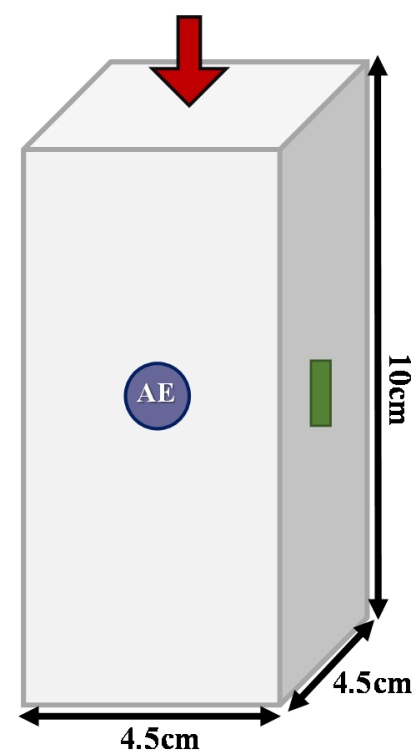

(a)

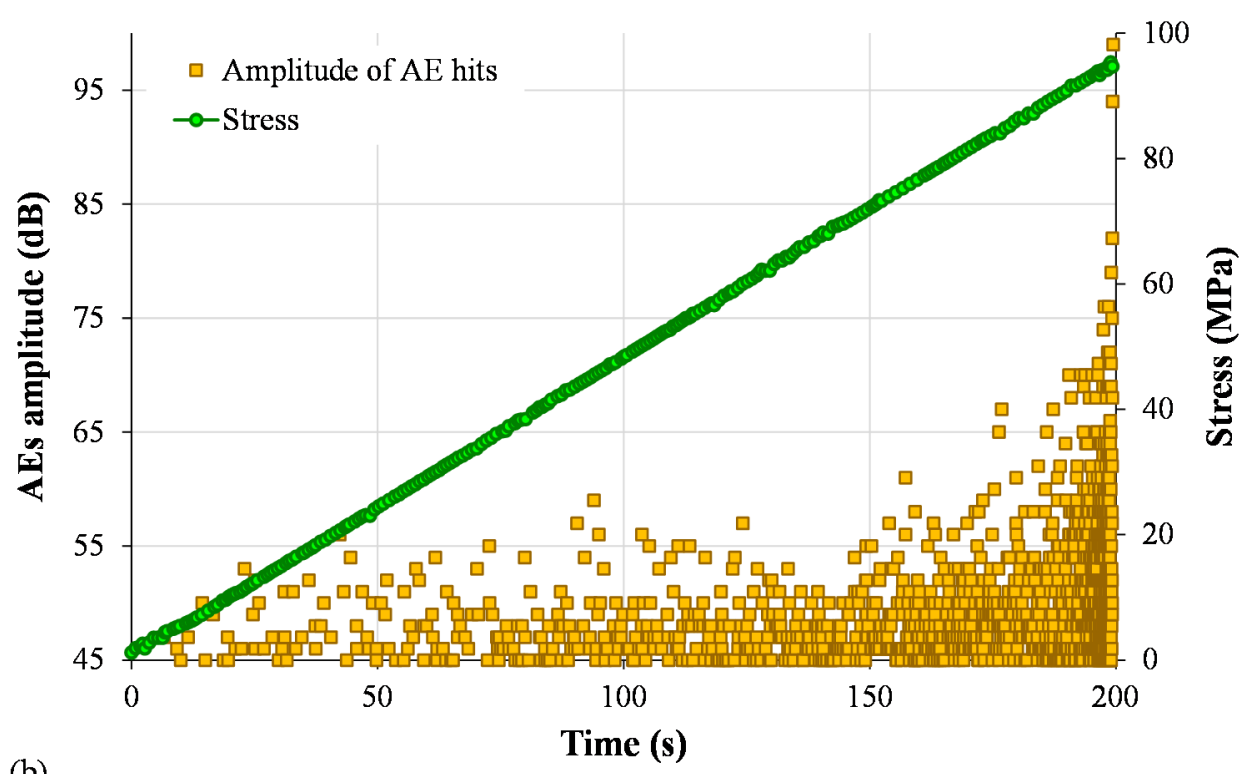

(b)

Figure 6. (a) Sketch of the intact prismatic specimen. The red arrow indicates the direction of the imposed load and the green rectangle the strain gauge. (b) The temporal evolution of the amplitudes of the acoustic hits in juxtaposition to that of the compressive force imposed.
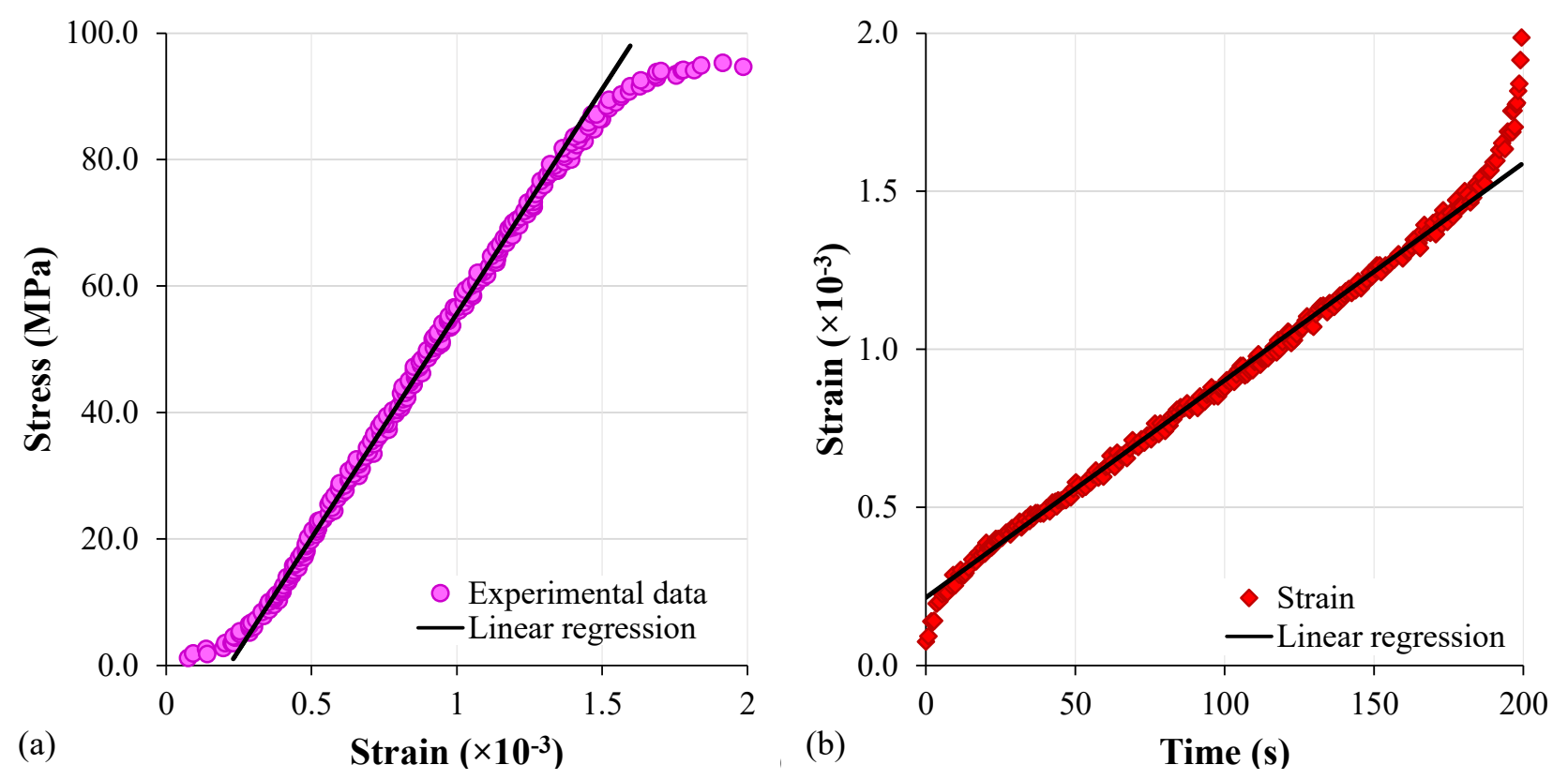

Figure 7. (a) The axial stress versus the axial strain for the intact prismatic marble specimen. (b) The temporal evolution of the strain for the intact prismatic marble specimen. 


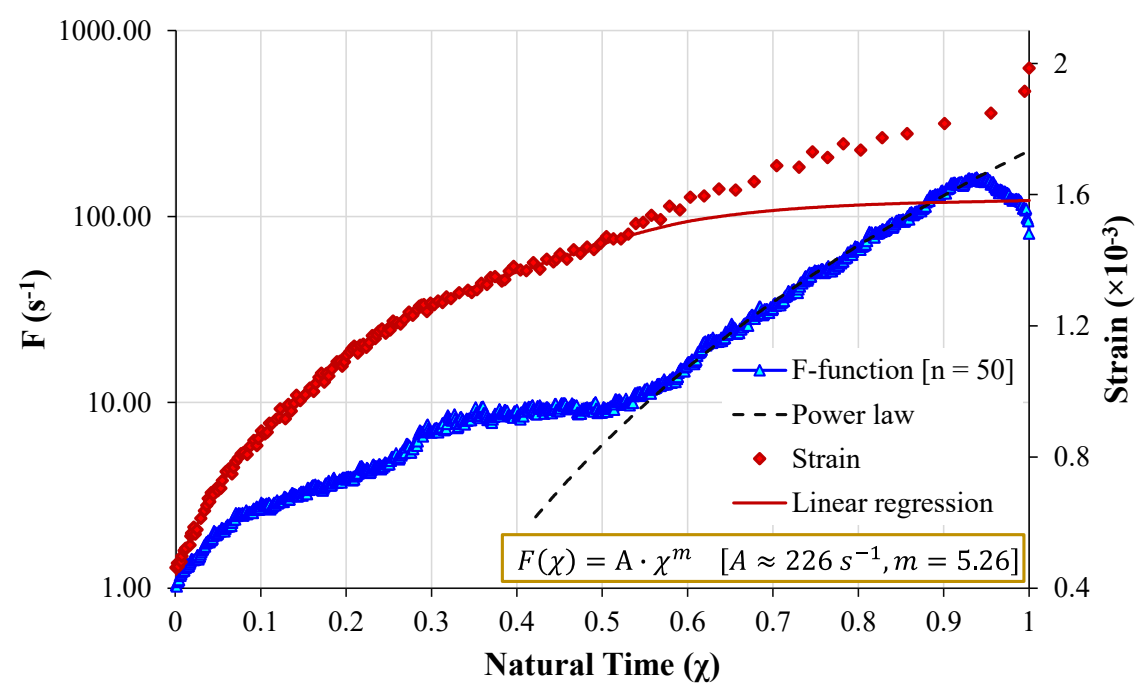

Figure 8. The evolution in the Natural Time domain of the F-function and the axial strain developed for the intact prismatic specimen under compression.

\subsection{Diametral Compression of Circular Semi Ring Marble Specimens}

In this protocol Circular Semi Rings (CSR) made of Dionysos marble were subjected to diametral compression until fracture. The geometry and the dimensions of the specimen along with the position of the acoustic sensor, from which the AE data were drawn, are shown in Figure 9a. Details about the specific protocol and the motive that dictated its introduction can be found in a series of publications by Kourkoulis et al. [44] and by Markides et al. [45]. The load was imposed with the aid of two metallic cylinders fitted into grooves of proper shape and size curved on the outer perimeter of the specimens. Displacement-control loading mode was adopted at a constant rate of $0.02 \mathrm{~mm} / \mathrm{min}$.
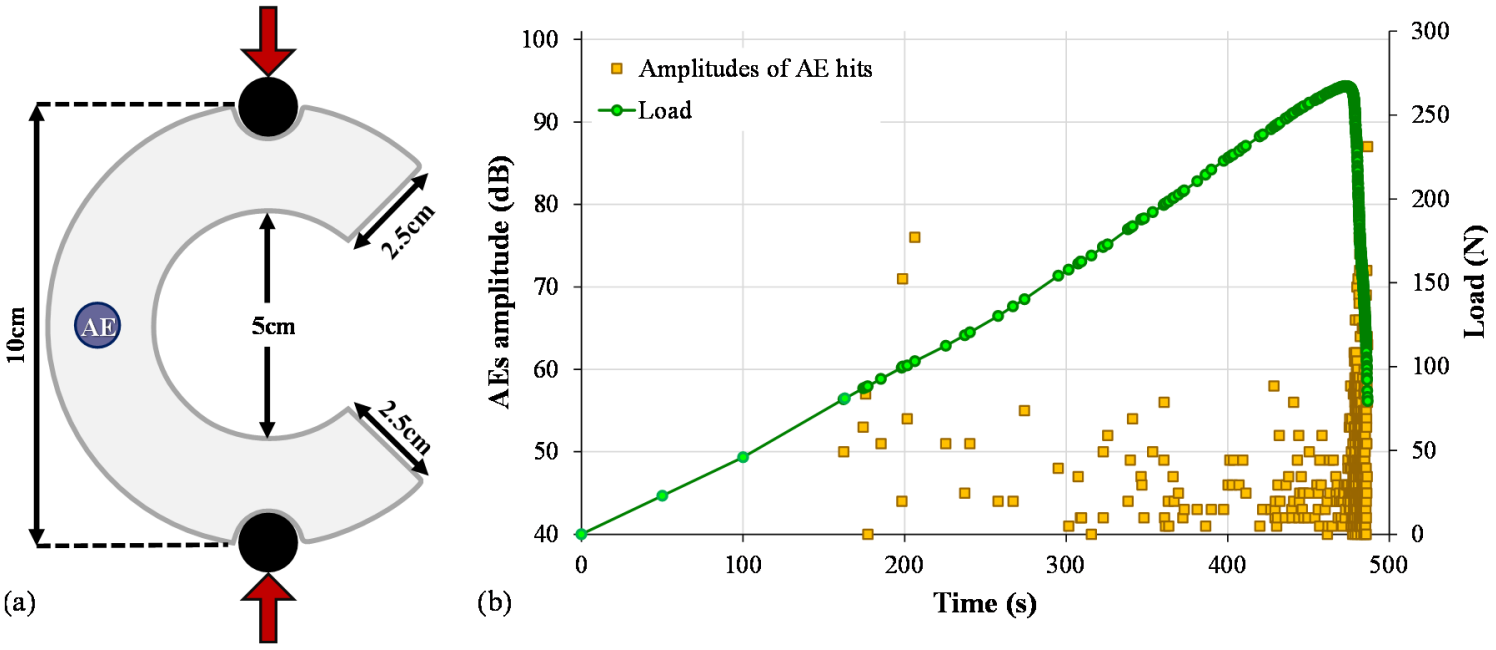

Figure 9. (a) The geometrical characteristics of the CSR marble specimen. The red arrows indicate the direction of the imposed force, and the black circles represent the cylinders used to apply the force. (b) The temporal evolution of the amplitudes of the acoustic hits in juxtaposition to that of the force.

During a representative experiment of this protocol, a total of about $N=507$ acoustic hits of amplitude exceeding $40 \mathrm{~dB}$ were recorded. The time evolution of the load imposed is plotted in Figure $9 \mathrm{~b}$ together with that of the amplitudes of the acoustic hits. It is worth mentioning that the specimen appears almost "silent" concerning its acoustic activity up to a time instant $t=162 \mathrm{~s}$, when the first $\mathrm{AE}$ was recorded. The load level at that instant 
was about $80 \mathrm{~N}$ or, equivalently, about $30 \%$ of the maximum value attained. The load was increasing up to the instant $t=473 \mathrm{~s}$ and then it started decreasing.

To calculate the $F$-function $n=15$ consecutive interevent time intervals were used. The evolution of the $F$-function in Natural Time is shown in Figure 10 in juxtaposition to the respective one of the loads applied, also in Natural Time. It is observed that when the load has exceeded its maximum value $(267 \mathrm{~N})$, the behavior of the function $F(\chi)$ obeys the power law of Equation (2), until the instant at which the load is reduced to $255 \mathrm{~N}$. Proper fitting of the data available indicates that during this interval, the exponent $m$ is equal to 6.62. The validity of the power law lasts from $\chi=0.21$ until $\chi=0.36$. It is here emphasized that for the specific experiment (and the remaining ones of this protocol) the majority of acoustic hits (more than $75 \%$ ) were detected after the maximization of the externally applied load.

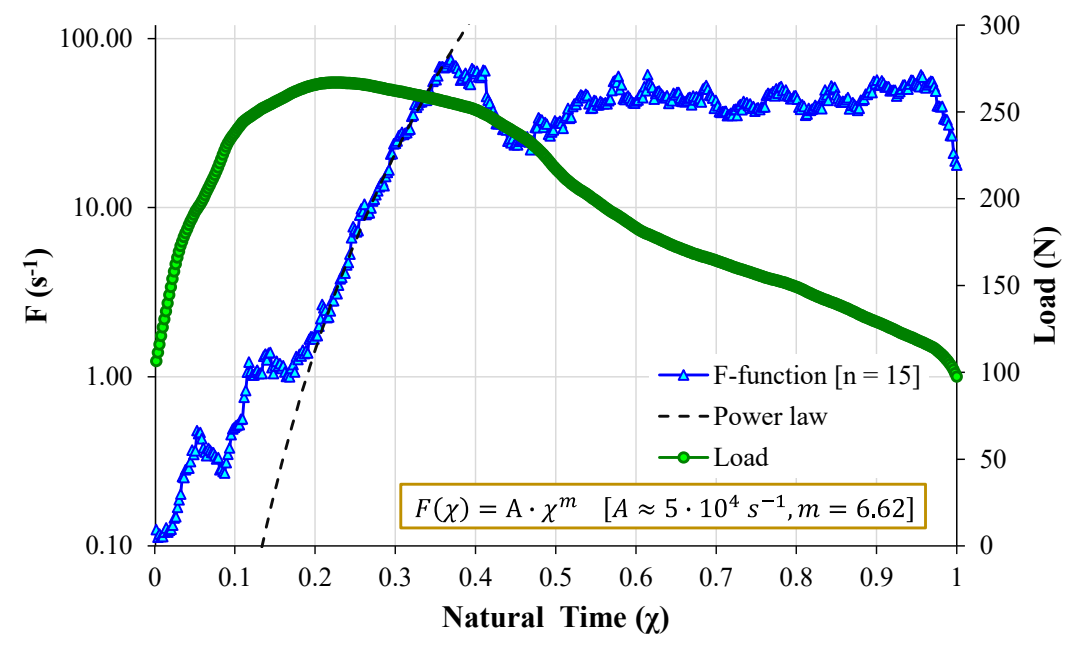

Figure 10. The evolution in the Natural Time domain of the F-function and of the load imposed for the CSR marble specimen.

\subsection{Shear of Mutually Interconnected Marble Blocks}

In this section data obtained from a structural experimental protocol during which complex structures consisting of two blocks of Dionysos marble, mutually interconnected by means of an "I" shaped titanium connector, were subjected to pure shear. The titanium connector is placed in grooves carved onto the marble blocks and is then completely covered with cementitious material (Figure 11a). A detailed description of the geometry of the structures (specimens) is given by Pasiou et al. [46]. The loading scheme intended to impose a system of forces on one of the two blocks so that it moves parallel to the other (rigidly clamped) block in order for the "I" connector to be under pure shear. Displacement-control loading mode was adopted at a constant rate equal to $0.2 \mathrm{~mm} / \mathrm{min}$. Eight sensors were used for detecting and recording the AE data. For the typical test studied here, catastrophic fracture (of the moving block) occurred when the force applied attained a maximum value of $27.5 \mathrm{kN}$. During the whole loading process $N=1092$ acoustic events were recorded. The temporal evolution of their amplitude is shown in Figure 11b, together with the respective evolution of the load imposed.

For the calculation of the $F$-function $n=30$ consecutive interevent time intervals were used. The evolution of the F-function in terms of conventional time is shown in Figure 12a, again, together with the evolution of the load applied. The sharp increase of the $F$-function is detected at the instant with $\tau \approx 860 \mathrm{~s}$ when the load attained a value of $27.2 \mathrm{kN}$, i.e., at about $99 \%$ of the respective maximum value reached during this experiment. In the previous time interval, i.e., for $\tau<860 \mathrm{~s}$, the $F$-function exhibits progressively increasing values with a series of local peaks, which are attributed to locally intense acoustic activity due to the formation of many microcracks at the interface between the titanium connector and the cementitious material and to friction between the two marble blocks and, also, between the marble blocks and the cementitious material. 


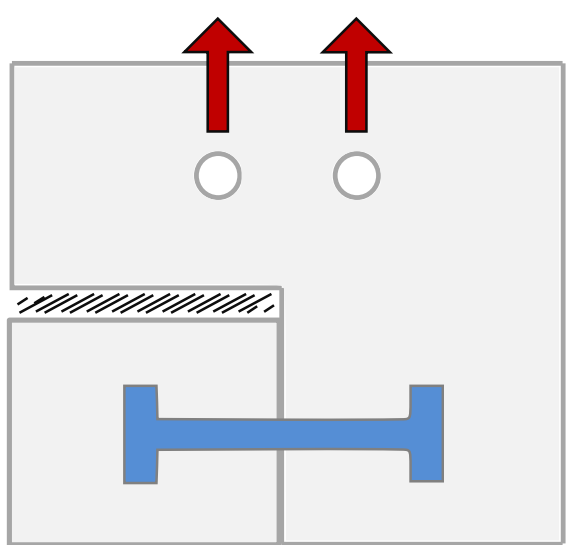

(a)

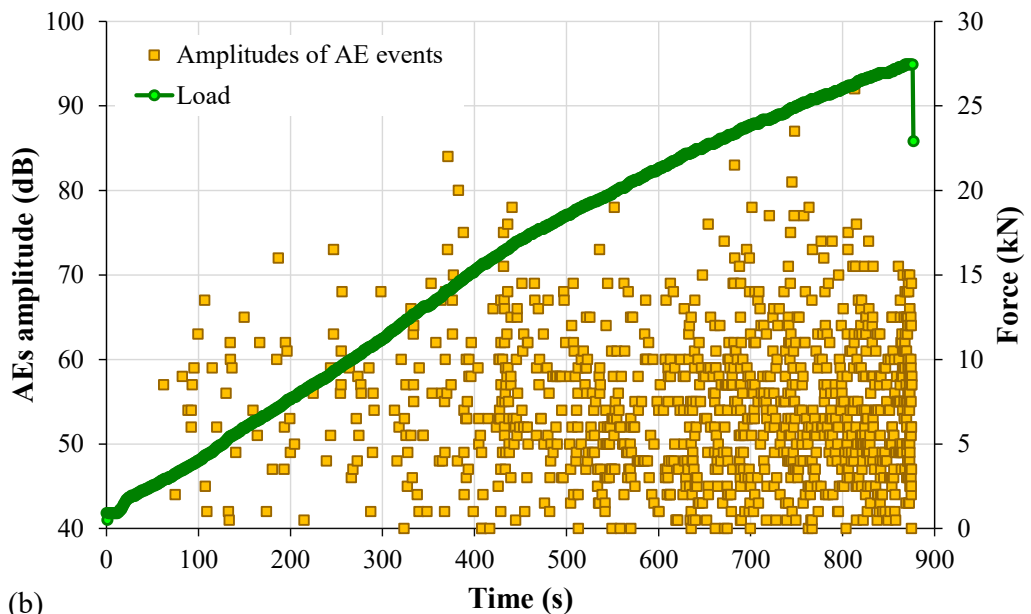

(b)

Time (s)

Figure 11. (a) Sketch of the mutually interconnected marble blocks. The red arrows indicate the direction of the imposed force. (b) The temporal evolution of the amplitudes of the acoustic events recorded in juxtaposition to the force imposed.

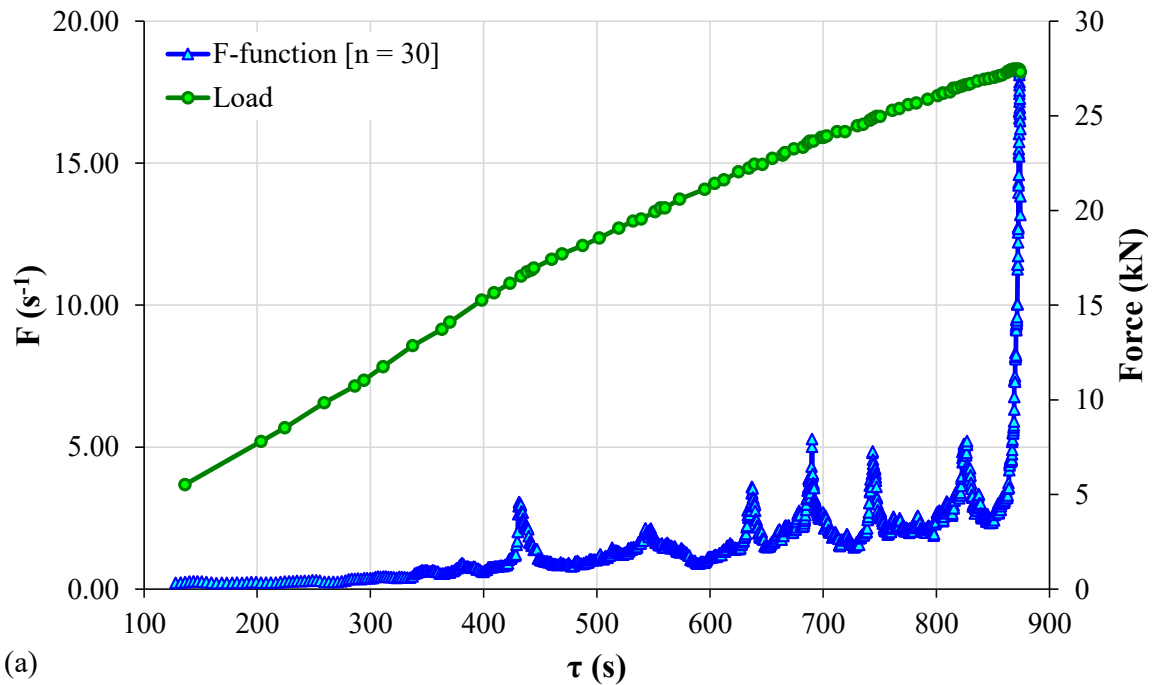

(a)

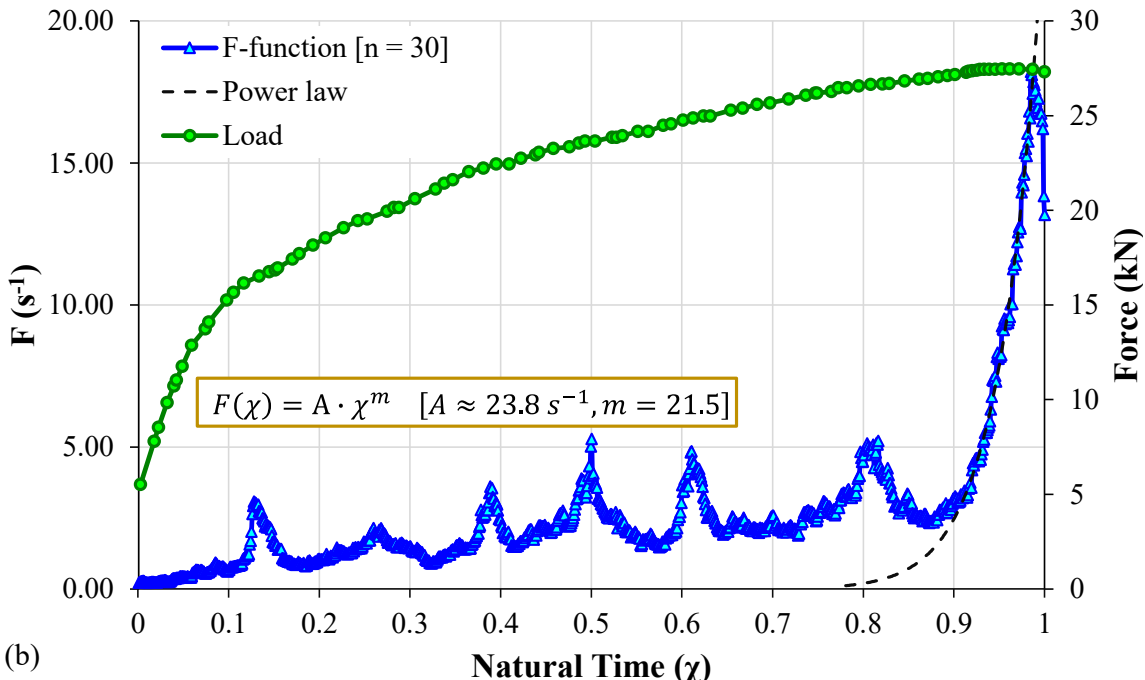

Figure 12. (a) The temporal evolution of the F-function and the load imposed. (b) The respective evolution in the Natural Time domain for the mutually interconnected marble blocks under shear. 
The evolution of the F-function in the Natural Time domain is shown in Figure $12 \mathrm{~b}$ in juxtaposition to the respective one of the imposed loads (also, in Natural Time terms). The existence of a power law governing the evolution of the $F(\chi)$ function is now observed when the applied load approaches its maximum value, and the catastrophic fracture is impending. The validity of the power law lasts from $\chi=0.91$ until $\chi=0.97$. By proper fitting, the exponent $m$ for the specific experiment was found equal to 21.5. This high value is well explained by the fact that a great number of acoustic events are produced in a quite short period of time, for the reasons mentioned above.

\section{Discussion and Conclusions}

The evolution of the F-function in the Natural Time domain was studied in a series of five experimental protocols, during which Dionysos marble specimens of various geometries were subjected to different loading schemes. Both elementary and structural protocols were considered. In spite of the wide variety of the protocols studied (in terms of the geometry of the specimens and the loading schemes imposed) the evolution of the F-function in the Natural Time domain exhibited a more or less self-consistent motive: The $F(\chi)$ values increase progressively for the major portion of the loading process, with some fluctuations, the number and intensity of which depend on the specific protocol. During the very last loading stages, i.e., while macroscopic fracture is approaching, the evolution of the $F(\chi)$ function is systematically governed by a power law of the form $F(\chi)=A \chi^{m}$. The characteristics of the domain that is governed by the above power law are briefly summarized in Table 1.

Table 1. The characteristics of the interval during which the $F$-function expressed in terms of the Natural Time concept is governed by a power law of the form: $F(\chi)=A \chi^{m}$.

\begin{tabular}{ccccc}
\hline Experiment & Natural Time & Load Region & $\begin{array}{c}\text { Maximum } \\
\text { Load }\end{array}$ & Exponent $\boldsymbol{m}$ \\
\hline Direct tension & {$[0.36-0.85]$} & {$[2931 \mathrm{~N}-2928 \mathrm{~N}]$} & $2933 \mathrm{~N}$ & 4.65 \\
Three-point bending & {$[0.46-0.79]$} & {$[647 \mathrm{~N}-643 \mathrm{~N}]$} & $656.9 \mathrm{~N}$ & 8.80 \\
Uniaxial compression & {$[0.58-0.93]$} & {$[89.9 \mathrm{MPa}-94.2 \mathrm{MPa}]$} & $95.3 \mathrm{MPa}$ & 5.26 \\
Diametral compression & {$[0.21-0.36]$} & {$[267 \mathrm{~N}-255 \mathrm{~N}]$} & $267 \mathrm{~N}$ & 6.62 \\
Shear & {$[0.91-0.97]$} & {$[27.25 \mathrm{kN}-27.45 \mathrm{kN}]$} & $27.5 \mathrm{kN}$ & 21.5 \\
\hline
\end{tabular}

Note: the blue color indicates a load value before it reaches its maximum value and the red color indicates the respective value after maximization of the load (stage of decreasing load).

It is concluded, therefore, that in case the acoustic activity, expressed in terms of the F-function, is explored in the Natural Time domain, important information is extracted regarding the proximity of the loaded system into its critical stage: The onset of validity of the above-mentioned power law provides a clear signal indicating that the system entered into the stage of impending macroscopic fracture.

The above conclusions are definitely supported by similar ones drawn by the analysis of the electric activity developed during all five protocols presented in this study. The temporal evolution of the electrical activity was here investigated by means of the pressure stimulated currents (PSC) technique [47], which is based on the detection and recording of weak electric currents, systematically produced during the mechanical loading of specific classes of brittle materials. This laboratory sensing technique is successfully applied worldwide, and it has been proven that it provides reliable pre-failure indicators $[19,42,48-51]$ in the form of a rapid increase of the intensity of the electric current while the specimens are about to enter into its critical stage, namely that of impending fracture.

Therefore, it was considered that it would be useful to compare the findings concerning the pre-failure indicator provided by the evolution of the F-function in the Natural Time domain against the respective ones provided by the PSC technique. In this direction, PSC data are here considered as obtained from the protocol with Circular Semi Rings under diametral compression. The temporal evolution of the PSC recorded during the specific experiment of this protocol discussed in Section 3.3 is plotted in Figure 13. 


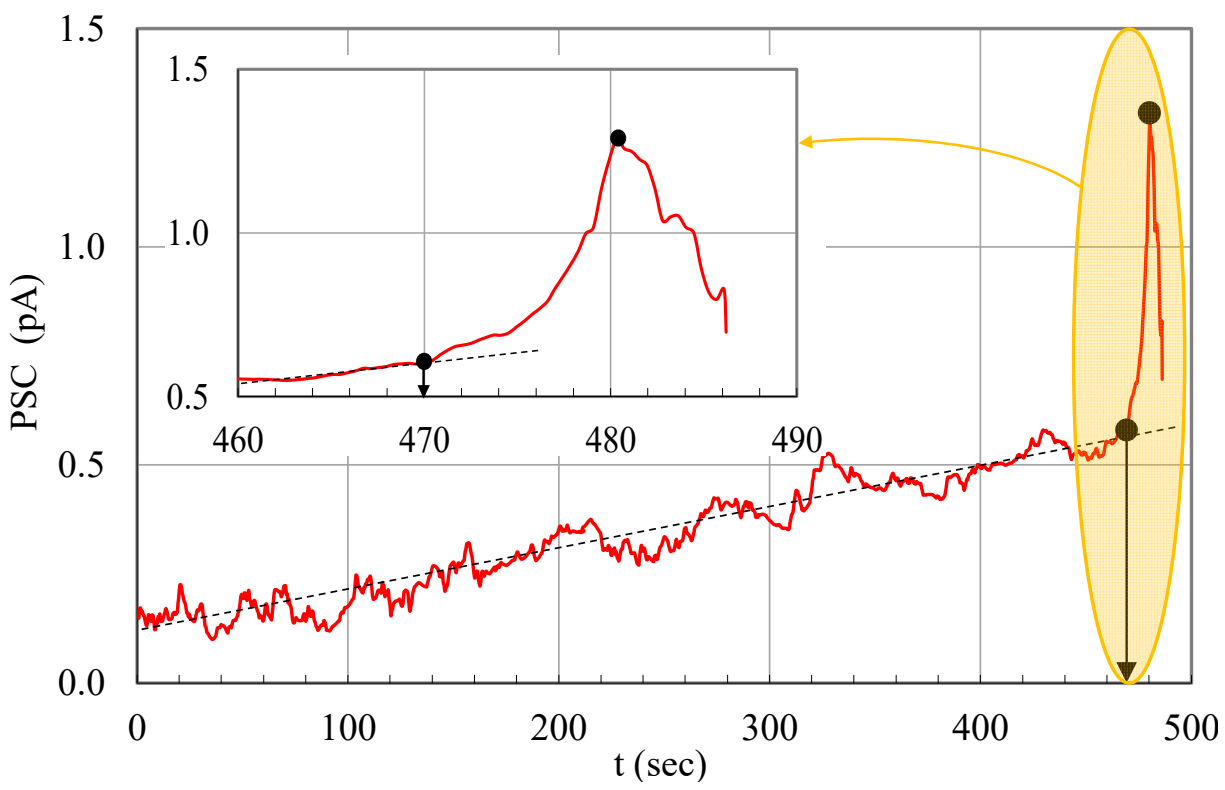

Figure 13. The temporal evolution of the PSC recorded during the diametral compression of the circular semi ring specimen discussed in Section 3.3.

It is seen from this figure that for the major portion of the test's duration the PSC increases smoothly and almost linearly with some rather smooth fluctuations. However, at the instant $t \approx 470 \mathrm{~s}$ the intensity of the PSC exhibits a rapid increase. The specific time instant, according to the data provided by the AEs, corresponds to a Natural Time instant with $\chi \approx 0.19$, which is exactly the instant at which the function $F(\chi)$ starts increasing rapidly obeying the power law of Equation (2) (see Figure 10).

Moreover, the PSC is maximized at the instant $t \approx 480 \mathrm{~s}$, which corresponds to $\chi \approx$ 0.46 , i.e., when the $F(\chi)$ function has already attained its maximum level. In other words, a clear direct analogy is observed between the intensification of the electric activity and the respective acoustic one as it is described by the F-function in the Natural Time domain.

The above conclusion is definitely supported by considering the electric current recorded during the protocol with mutually interconnected marble blocks under shear, discussed in Section 3.4. The respective evolution of the PSC is plotted in Figure 14.

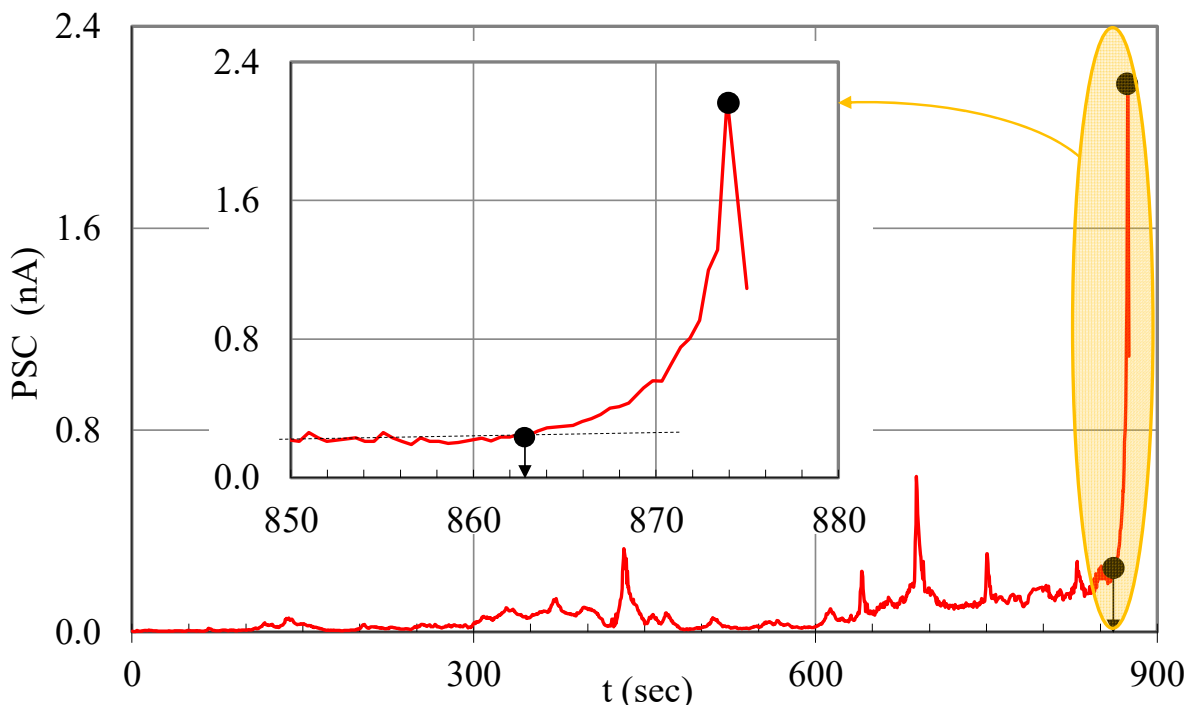

Figure 14. The temporal evolution of the PSC recorded during the experiment with mutually interconnected marble blocks under pure shear, that was discussed in Section 3.4. 
It is seen that the strong intensification of the electric activity is detected at the instant $t \approx 865$ s, corresponding to $\chi \approx 0.91$, which is again the instant at which the function $F(\chi)$ starts increasing rapidly obeying the power law of Equation (2) (see Figure 12b). Similarly, the PSC attains its peak value at $t \approx 874 \mathrm{~s}$ which corresponds to $\chi \approx 0.985$, the instant at which the F-function, in terms of the Natural Time domain has, also, attained its peak value.

Quite similar conclusions concerning the temporal evolution of the PSC and its close agreement with the respective one of the F-function analyzed in the Natural Time domain are drawn from the electric activity recorded during the remaining protocols of this study. Clearly, further research is needed with additional experimental protocols and a wider range of materials. In any case, the conclusions of this preliminary study are encouraging, indicating, at least, that the topic deserves further attention.

Author Contributions: Conceptualization, D.T.; methodology, D.T. and A.L.; experimental, E.D.P., S.K.K., A.L. and I.S.; writing-original draft preparation, D.T.; writing-review and editing, S.K.K., A.L., E.D.P. and I.S.; visualization, S.K.K.; supervision, D.T. and S.K.K.; project administration, D.T. All authors have read and agreed to the published version of the manuscript.

Funding: This research received no external funding.

Institutional Review Board Statement: Not applicable.

Informed Consent Statement: Not applicable.

Data Availability Statement: Not applicable.

Conflicts of Interest: The authors declare no conflict of interest.

\section{References}

1. Kishinouye, F. An experiment on the progression of fracture. (A preliminary report). Jisin 1934, 6, 24-31, translated and published by Ono K. J. Acoust. Emiss. 1990, 9, 177-180.

2. Förster, F. Akustische Untersuchung der Bildung von Martensitnadeln (Acoustic Study of the Formation of Martensile Needels). Z. Für Met. 1936, 29, 245.

3. Kaiser, E. A Study of Acoustic Phenomena in Tensile Test. Ph.D. Thesis, Technical University of Munich, Munich, Germany, 1950.

4. Grosse, C.; Ohtsu, M. (Eds.) Acoustic Emission Testing; Springer: Berlin/Heidelberg, Germany, 2008.

5. Sagar, R.V.; Prasad, B.K.R. A review of recent developments in parametric based acoustic emission techniques applied to concrete structures. Nondestruct. Test. Eval. 2012, 27, 47-68. [CrossRef]

6. Rao, M.V.M.S.; Lakshmi, K.J.P. Analysis of b-value and improved b-value of acoustic emissions accompanying rock fracture. Curr. Sci. 2005, 89, 1577-1582.

7. Sagar, R.V.; Prasad, B.K.R.; Kumar, S.S. An experimental study on cracking evolution in concrete and cement mortar by the b-value analysis of acoustic emission technique. Cem. Concr. Res. 2012, 42, 1094-1104. [CrossRef]

8. Aggelis, D.G.; Soulioti, D.V.; Sapouridis, N.; Barkoula, N.M.; Paipetis, A.S.; Matikas, T.E. Acoustic emission characterization of the fracture process in fibre reinforced concrete. Constr. Build. Mater. 2011, 25, 4126-4131. [CrossRef]

9. Loukidis, A.; Triantis, D.; Stavrakas, I.; Pasiou, E.D.; Kourkoulis, S.K. Comparative Ib-value and F-function analysis of Acoustic Emissions from elementary and structural tests with marble specimens. Mater. Des. Process. Commun. 2021, 3, e176. [CrossRef]

10. Triantis, D.; Kourkoulis, S.K. An alternative approach for representing the data provided by the acoustic emission technique. Rock Mech. Rock Eng. 2018, 51, 2433-2438. [CrossRef]

11. Zhang, J.-Z.; Zhou, X.-P.; Zhou, L.-S.; Berto, F. Progressive failure of brittle rocks with non-isometric flaws: Insights from acousto-optic-mechanical (AOM) data. Fatigue Fract. Eng. Mater. Struct. 2019, 42, 1787-1802. [CrossRef]

12. Wang, X.; Wang, E.; Liu, X. Damage characterization of concrete under multi-step loading by integrated ultrasonic and acoustic emission techniques. Constr. Build. Mater. 2019, 221, 678-690. [CrossRef]

13. Saltas, V.; Peraki, D.; Vallianatos, F. The use of acoustic emissions technique in the monitoring of fracturing in concrete using soundless chemical demolition agent. Frat. Integrità Strutt. 2019, 13, 505-516. [CrossRef]

14. Yao, W.; Yu, J.; Liu, X.; Zhou, X.; Cai, Y.; Zhu, Y. Study on acoustic emission characteristics and failure prediction of post-hightemperature granite. J. Test. Eval. 2019, 48, 2459-2473. [CrossRef]

15. Ge, Z.; Sun, Q. Acoustic emission characteristics of gabbro after microwave heating. Int. J. Rock Mech. Min. Sci. 2021, 138, 104616. [CrossRef]

16. Zhou, X.; Niu, Y.; Cheng, H.; Berto, F. Cracking behaviors and chaotic characteristics of sandstone with unfilled and filled dentate flaw. Theor. Appl. Fract. Mech. 2021, 112, 102876. [CrossRef] 
17. Huang, J.; Liao, Z.; Hu, Q.; Song, Z.; Wang, X. Fracture mechanism of tight sandstone under high and complex 3-D stress compression: Insights from acoustic emission. J. Pet. Sci. Eng. 2022, 208, 109635. [CrossRef]

18. Triantis, D.; Tsaousi, D.K.; Stavrakas, I.; Pasiou, E.D.; Douvis, P.; Kourkoulis, S.K. Electric and acoustic activity in notched fiber-reinforced concrete beams under three-point bending. Mater. Today Proc. 2020, 32, 148-155. [CrossRef]

19. Triantis, D.; Pasiou, E.D.; Stavrakas, I.; Kourkoulis, S.K. Hidden affinities between electric and acoustic activity in brittle materials at near-fracture load levels. Rock Mech. Rock Eng. 2021. to appear. [CrossRef]

20. Triantis, D.; Stavrakas, I.; Loukidis, A.; Pasiou, E.D.; Kourkoulis, S.K. Exploring the acoustic activity in marble specimens under tension while entering into the stage of impending fracture. Procedia Struct. Integr. 2021, 33, 330-336. [CrossRef]

21. Bak, P.; Tang, C.; Wiesenfeld, K. Self-organized criticality: An explanation of the 1/f noise. Phys. Rev. Lett. 1987, 59, 381-384. [CrossRef] [PubMed]

22. Sarlis, N.V.; Varotsos, P.A.; Skordas, E.S. Flux avalanches in $\mathrm{YBa}_{2} \mathrm{Cu}_{3} \mathrm{O}_{7-\mathrm{x}}$ films and rice piles: Natural time domain analysis. Phys. Rev. B 2006, 73, 054504. [CrossRef]

23. Varotsos, P.; Sarlis, N.V.; Skordas, E.S.; Uyeda, S.; Kamogawa, M. Natural time analysis of critical phenomena. Proc. Natl. Acad. Sci. USA 2011, 108, 11361-11364. [CrossRef]

24. Varotsos, P.A.; Sarlis, N.V.; Skordas, E.S. Spatio-temporal complexity aspects on the interrelation between seismic electric signals and seismicity. Pract. Athens Acad. 2001, 76, 294-321.

25. Varotsos, P.A.; Sarlis, N.V.; Skordas, E.S. Long-range correlations in the electric signals that precede rupture. Phys. Rev. E Stat. Nonlin. Soft Matter Phys. 2002, 66, 011902. [CrossRef] [PubMed]

26. Varotsos, P.A.; Sarlis, N.V.; Skordas, E.S. Natural Time Analysis: The New View of Time: Precursory Seismic Electric Signals, Earthquakes and Other Complex Time Series; Springer Science \& Business Media: Berlin/Heidelberg, Germany, 2011.

27. Varotsos, P.A.; Sarlis, N.V.; Tanaka, H.K.; Skordas, E.S. Similarity of fluctuations in correlated systems: The case of seismicity. Phys. Rev. E 2005, 72, 041103. [CrossRef] [PubMed]

28. Varotsos, P.A.; Sarlis, N.V.; Skordas, E.S.; Tanaka, H.K.; Lazaridou, M.S. Entropy of seismic electric signals: Analysis in natural time under time reversal. Phys. Rev. E 2006, 73, 031114. [CrossRef] [PubMed]

29. Uyeda, S.; Kamogawa, M.; Tanaka, H. Analysis of electrical activity and seismicity in the natural time domain for the volcanicseismic swarm activity in 2000 in the Izu Island region, Japan. J. Geophys. Res. Solid Earth 2009, 114. [CrossRef]

30. Vallianatos, F.; Michas, G.; Papadakis, G. Non-extensive and natural time analysis of seismicity before the Mw6.4, October 12, 2013 earthquake in the South West segment of the Hellenic Arc. Phys. A Stat. Mech. Its Appl. 2014, 414, 163-173. [CrossRef]

31. Varotsos, P.A.; Sarlis, N.V.; Skordas, E.S.; Lazaridou, M.S. Identifying sudden cardiac death risk and specifying its occurrence time by analyzing electrocardiograms in natural time. Appl. Phys. Lett. 2007, 91, 064106. [CrossRef]

32. Baldoumas, G.; Peschos, D.; Tatsis, G.; Votis, C.I.; Chronopoulos, S.K.; Christofilakis, V.; Kostarakis, P.; Sarlis, N.V.; Skordas, E.S.; Naka, K.K.; et al. Comparison of the R-R intervals in ECG and Oximeter signals to be used in complexity measures of Natural Time Analysis. In Proceedings of the 2018 7th International Conference on Modern Circuits and Systems Technologies (MOCAST), Thessaloniki, Greece, 7-9 May 2018.

33. Sarlis, N.V.; Skordas, E.S.; Varotsos, P.A. Similarity of fluctuations in systems exhibiting Self-Organized Criticality. EPL (Europhys. Lett.) 2011, 96, 28006. [CrossRef]

34. Vallianatos, F.; Michas, G.; Benson, P.; Sammonds, P. Natural time analysis of critical phenomena: The case of acoustic emissions in triaxially deformed Etna basalt. Phys. A Stat. Mech. Its Appl. 2013, 392, 5172-5178. [CrossRef]

35. Hloupis, G.; Stavrakas, I.; Pasiou, E.D.; Triantis, D.; Kourkoulis, S.K. Natural Time Analysis of Acoustic Emissions in Double Edge Notched Tension (DENT) Marble Specimens. Procedia Eng. 2015, 109, 248-256. [CrossRef]

36. Loukidis, A.; Pasiou, E.D.; Sarlis, N.V.; Triantis, D. Fracture analysis of typical construction materials in natural time. Phys. A Stat. Mech. Its Appl. 2020, 547, 123831. [CrossRef]

37. Tsuji, D.; Katsuragi, H. Temporal analysis of acoustic emission from a plunged granular bed. Phys. Rev. E 2015, $92,042201$.

38. Niccolini, G.; Lacidogna, G.; Carpinteri, A. Fracture precursors in a working girder crane: AE natural-time and b-value time series analyses. Eng. Fract. Mech. 2019, 210, 393-399. [CrossRef]

39. Niccolini, G.; Potirakis, S.M.; Lacidogna, G.; Borla, O. Criticality hidden in acoustic emissions and in changing elec-trical resistance during fracture of rocks and cement-based materials. Materials 2020, 13, 5608. [CrossRef]

40. Friedrich, L.F.; Cezar, É.S.; Colpo, A.B.; Tanzi, B.N.R.; Sobczyk, M.; Lacidogna, G.; Niccolini, G.; Kosteski, L.E.; Iturrioz, I. Long-Range Correlations and Natural Time Series Analyses from Acoustic Emission Signals. Preprints 2021. not peer-reviewed. [CrossRef]

41. Kourkoulis, S.K.; Pasiou, E.D.; Dakanali, I.; Stavrakas, I.; Triantis, D. Notched marble plates under direct tension: Mechanical response and fracture. Constr. Build. Mater. 2018, 167, 426-439. [CrossRef]

42. Kourkoulis, S.K.; Pasiou, E.D.; Dakanali, I.; Stavrakas, I.; Triantis, D. Notched marble plates under tension: Detecting pre- failure indicators and predicting entrance to the "critical stage". Fatigue Fract. Eng. Mater. Struct. 2018, 41, 776-786. [CrossRef]

43. Perdikatsis, V.; Kritsotakis, K.; Markopoulos, T.; Laskaridis, K. Discrimination of Greek marbles by trace-, isotope-and mineralogical analysis. In Fracture and Failure of Natural Building Stones; Kourkoulis, S.K., Ed.; Springer: Dordrecht, The Netherlands, 2007; pp. 497-515. [CrossRef]

44. Kourkoulis, S.K.; Pasiou, E.D.; Markides, C.F. Analytical and numerical study of the stress field in a Circular Semi Ring under combined diametral compression and bending. Frat. Ed. Integrità Strutt. 2018, 13, 247-265. [CrossRef] 
45. Markides, C.F.; Stavropoulou, M.; Pasiou, E.D.; Kourkoulis, S.K. Enlightening the role of critical parameters for the determination of the tensile strength by means of the Circular Semi Ring test. Procedia Struct. Integr. 2020, 25, 214-225. [CrossRef]

46. Pasiou, E.D.; Stavrakas, I.; Triantis, D.; Kourkoulis, S.K. Marble epistyles under shear: An experimental study of the role of "Relieving Space". Front. Struct. Civ. Eng. 2019, 13, 767-786. [CrossRef]

47. Triantis, D.; Stavrakas, I.; Anastasiadis, C.; Kyriazopoulos, A.; Vallianatos, F. An analysis of pressure stimulated currents (PSC), in marble samples under mechanical stress. Phys. Chem. Earth Parts A/B/C 2006, 31, 234-239. [CrossRef]

48. Kyriazopoulos, A.; Anastasiadis, C.; Triantis, D.; Brown, C.J. Non-destructive evaluation of cement-based materials from pressure-stimulated electrical emission-Preliminary results. Constr. Build. Mater. 2011, 25, 1980-1990. [CrossRef]

49. Li, Z.; Wang, E.; He, M. Laboratory Studies of Electric Current Generated during Fracture of Coal and Rock in Rock Burst Coal Mine. J. Min. 2015, 235636. [CrossRef]

50. Cartwright-Taylor, A.; Vallianatos, F.; Sammonds, P. Superstatistical view of stress-induced electric current fluctuations in rocks. Phys. A Stat. Mech. Its Appl. 2014, 414, 368-377. [CrossRef]

51. Li, D.X.; Wang, E.Y.; Li, Z.H.; Ju, Y.Q.; Wang, D.M.; Wang, X.Y. Experimental investigations of pressure stimulated currents from stressed sandstone used as precursors to rock fracture. Int. J. Rock Mech. Min. Sci. 2021, 145, 104841. [CrossRef] 